\title{
MAG-2 promotes invasion, mobility and adherence capability of lung cancer cells by MMP-2, CD44 and intracellular calcium in vitro
}

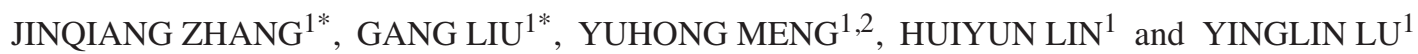 \\ ${ }^{1}$ Institute of Basic Medical Sciences, No. 27 Taiping Road, Haidian District, Beijing 100850; ${ }^{2}$ Department of \\ Pathology, General Navy Hospital, No. 6 Fucheng Road, Haidian District, Beijing 100037, P.R. China
}

Received November 19, 2008; Accepted December 30, 2008

DOI: $10.3892 /$ or_00000274

\begin{abstract}
Tumor metastasis, the important characteristic of malignant tumors, is closely associated with a series of changes in the expressions of genes and proteins. A novel gene MAG-2, which may have close correlation with lung cancer metastasis, was identified in our laboratory through an approach of suppressed subtractive hybridization using lung cancer cell strains with the same origin but different metastatic potential as models. The relations between MAG-2 gene and aspects of cancer metastasis including invasion, mobility, anchorageindependent growth capability and adherence to ECM, were investigated in our experiment models. MAG-2 gene was proved to be genuine and have mRNA and deduced proteins from itself by methods of expression profile analysis and fluorescence staining. Cytological experiments had demonstrated that hyper- or hypo-expressing of MAG-2 by gene transfection or RNAi leads to significant increase or decrease in the metastatic ability of cancer cells. In addition, CD44, MMP-2 and free calcium ion concentration intracellularly, were proved to be metastasis promoting factors, and found to be regulated by MAG-2 in lung cancer cells, this might be the mechanism of the metastasis promoting function of MAG-2 gene. The positive rate of MAG-2 mRNA was found to be significantly higher in tumor tissue from patients with metastatic lung cancer than tissues from patients with non-metastatic lung cancer. These data suggest that MAG-2 may be a novel causal gene for lung cancer invasion and metastasis.
\end{abstract}

\section{Introduction}

Metastasis is a multistage process, involving cancer cell motility, intravasation, transit in the blood, extravasation and

Correspondence to: Dr Yinglin Lu, Institute of Basic Medical Sciences, No. 27 Taiping Road, Haidian District, Beijing 100850, P.R. China

E-mail: luyinglin@hotmail.com

${ }^{*}$ Contributed equally

Key words: neoplasm metastasis, CD44, MMP-2, lung cancer, calcium growth at a new site (1). Many genes were found to be involved in each step of this lethal process of malign tumor (2). It is well known that cancer is a disease of extreme heterogeneity (3). Apparently, there are several alternative genetic paths which cells can take en route to forming a primary tumor. Thus, a particular phenotype e.g. metastasis required early in tumorigenesis by an evolving tumor cell can be acquired through the mutation of any one of several alternative growth-controlling genes (4). That means cancer cells of the same nest may have immense differences in their behavior, such as capability of clone forming, motility and invasion. To identify the causative genes under a certain phenotype would require to decipher the characteristics of the tumor $(5,6)$.

Phenotype cloning techniques have the potentials to isolate genes by virtue of their effect alone without requiring prior knowledge of their biochemical function or map position, and to save a great deal of repetitive work involving analysis of multiple markers (7), the Suppression Subtractive hybridization (SSH) is one of the methods of phenotype cloning with high efficiency (8). By employing the methods of SSH, two cDNA libraries were acquired in our previous study (9). The model cells used in the experiments were PLA801C and D, two human giant-cell lung carcinoma cell strains. Both were derived from the same parental cell line PLA801 and have almost the same karyotype except some differences on chromosome 7, 17, 18 and $\mathrm{X}$, and the most apparent diversity of the two sister cell strains comes from the capability of metastasis in vivo, D can form distance metastatic lesions in Xenograft mice while $C$ can not (10-12).

After SSH, all the cDNA segments of libraries were spoted as a microarray and hybridized with equally mixed cDNA fluorescence probes from both model cells. It was proved by microarray that the bulks of library fragments (and the homologous mRNA) have different copy numbers between $\mathrm{C}$ and $\mathrm{D}$ cell strain, which means the corresponding genes to those cDNA segments in libraries have distinct diversity on transcription activity in the two cell strains, and the expressional difference of these genes could contribute to metastatic discrepancy of PLA801D and C cells. The MAG-2 (metastasis associated gene-2) is one of these homologous genes and its transcript was identical with the GenBank recorded sequence BC002420.1. The transcriptional activity 
of MAG-2 in D cell strains was much higher than that in C cell strain, which was reconfirmed by Northern blotting (13).

In the present study, we investigated the relationship between the metastastic characteristics of lung cancer PLA801 cells and the gene expression of MAG-2. We demonstrated that overexpression of MAG-2 will elevate cell invasion, mobility and the capability of adherence to ECM (extracellular matrix, ECM), whereas those abilities were partly depressed if MAG-2 expression was inhibited. We also demonstrated that MAG-2 could promote the quantity of CD44, enhance the activation of MMP-2 and increase the concentration of intracellular free calcium.

Furthermore, we found that the detection rate of MAG-2 mRNA of patients with proven distant organ metastasis was much higher than that of patients without metastasis lesions. These results suggest that MAG-2 have the function of prometastasis, and the MMP-2, CD44 and calcium are involved in this process of metastasis regulation. These findings are the first to link the novel function-unknown gene with cell invasion and cell-matrix interaction, and will give clues to further exploration of MAG-2.

\section{Material and methods}

Cell lines and lung cancer samples. The human giant-cell lung carcinoma cells PLA801C and D were preserved in our laboratory, HepG2 cells were purchased from ATCC. PLA801 and HepG2 cells were maintained in RPMI-1640 and Dulbecco's modified Eagle's medium (Invitrogen, Carlsbad, CA, USA) respectively, and supplemented with $10 \%$ heat-inactivated fetal bovine serum (Hyclone, Logan, UT, USA), $100 \mathrm{U} / \mathrm{ml}$ penicillin and $100 \mu \mathrm{g} / \mathrm{ml}$ streptomycin, and incubated in a humidified incubator with $5 \% \mathrm{CO}_{2}$ at $37^{\circ} \mathrm{C}$. All the 24 cases of lung cancer tissues were obtaibed from Sample Bank of Beijing Cancer Hospital, with or without overt distance metastasis. Informed consents were obtained from all the patients enrolled in the Sample Bank.

Immunofluorescent staining. Procedures for physiological localization study of endogenous MAG-2 protein intracellularly were carried out as described previously (14) with slight modification. Briefly, PLA801D seeded on coverslips were rinsed 3 times in phosphate-buffered saline (PBS) and fixed in $4 \%$ paraformaldehyde for $15 \mathrm{~min}$, followed by permeabilization in $0.2 \%$ Triton X-100 for $15 \mathrm{~min}$. Then, cells were blocked with $1 \%$ BSA in PBS and incubated with diluted anti-MAG-2 polyclone antibody, produced in our laboratory by immunized rabbit with synthesized 17-aminoacid polypeptide of MAG-2 (15), at $4^{\circ} \mathrm{C}$ overnight, after washing, FITC-linked second antibody (Santa Cruz Biotech, Santa Cruz, CA, USA) was loaded at room temperature for $1 \mathrm{~h}$. Finally, cells were counter dyed by 4',6-diamidino-2phenylindole (Sigma, St. Louis, MO, USA) and observed under laser scanning confocal microscope (Radiance 2100, Bio-Rad Corporation, Hercules, CA, USA) at the excitation wavelength of $490 \mathrm{~nm}$.

RNA dot hybridization. RNA dot hybridization was performed using multiple tissue expression array 3.0 (Clontech, Mountain View, CA, USA), which is a positively charged nylon mem- brane spotted with 75 poly A+ RNAs from different human tissues. ${ }^{32} \mathrm{P}$ radioactive probes were synthesized by Klenow DNA polymerase with the MAG-2 cDNA as template. Hybridization was carried out in 50\% formamide, 6X SSC, $0.5 \%$ SDS, $5 \mathrm{X}$ Danhardt reagent and $100 \mu \mathrm{g} / \mathrm{ml}$ salmon sperm DNA at $42^{\circ} \mathrm{C}$ for $16 \mathrm{~h}$, then washed nylon membrane was autoradiographed at $-70^{\circ} \mathrm{C}$. The spots on exposed film were scanned and analyzed using Tanon GIS gel imaging system (Bio-Tanon Co., Ltd., Shanghai, P.R. China).

MAG-2 hyper- or hypo-expression in PLA801C or D strain, respectively. Sense and anti-sense expression vectors were constructed by inserting the MAG-2 full-length cDNA in different direction in the BamHI multi-cloning site of pcDNA3.0 plasmid (Invitrogen), the inserted sequence was the longer segment of pOBT7/BC002420 (Open Biosystem, Huntsville, AL, USA) plasmid after being digested by the endonuclease $\mathrm{BamHI}$, which contained the identical sequence with the only MAG-2 transcript. The proper inserting direction of sense and anti-sense vectors, termed as pcDNA3.0/MAG2S and AS, was conformed by DNA sequence analysis. Then, the cell strains PLA801C and D were transfected with pcDNA3.0/ MAG2S (C strain) and AS (D strain) respectively by Lipofectamin 2000 (Invitrogen) and pcDNA3.0 was used as control. RT-PCR assay was used to analyze the mRNA level of MAG-2 in stable transfected cells.

RNA interference. To create the small interfering RNA (siRNA) plasmid constructs, complementary strands of three oligonucleotides, MS1, MS2 and MS3 specifically targeting MAG-2 were synthesized. Oligo MS1: 5'-GATCCGTCC AGCTCAGTTCTGCGTTCTCAAGAGAAACGCAGAAC TGAGCTGGATTTTTTGGAAA-3'; Oligo MS2: 5'-GATCC ACACGACAAGAGGAAGCGTTTCAAGAGAACGCTTC CTCTTGTCGTGTTTTTTTGGAAA-3' Oligo MS3: 5'-GAT CCACCTTGTTGCGGCTCTCATTTCAAGAGAATGAGA GCCGCAACAAGGTTTTTTTGGAAA-3'. After annealing, double-stranded oligonucleotides were cloned into BamHI and HindIII linearized pSilencer 2.1-U6 vectors (Ambion Corporation, Cambridge, MA, USA). The inserts were confirmed by DNA sequence analysis. Stable transfection of PLA801D cell strain with each of the three reconstructions or control vector, that is pSilencer 2.1-U6/MS1, pSilencer 2.1-U6/MS2, pSilencer 2.1-U6/MS3 or pSilencer 2.1-U6 (empty vector) using Lipofectamin 2000 transfection reagent (Invitrogen) was carried out as recommended by the manufacturer.

Reverse transcriptase-polymerase chain reaction analysis. Total cellular RNA from cell lines or cancer tissues were extracted using TRIzol (Invitrogen) according to the manufacturer's protocol. Total RNA (5 $\mu \mathrm{g})$ was reverse transcripted using RT-PCR kit (Promega BioSciences, San Luis Obispo, CA, USA) as per the standard protocol and the primers used for PCR were synthesized as follow, MAG-2: forward, 5'AGAAAACAGCATTGGCCTTG-3', reverse 5'-TGGAGC ATCAGGGGGTTTT-3'; GAPDH: forward 5'-ACCACAGT CCATGCCATCAC-3', reverse 5'-TCCACCACCCTGT TGCTGTA-3'. The DNA were amplified under following conditions: $95^{\circ} \mathrm{C}$ for $5 \mathrm{~min}, 25$ cycles at $95^{\circ} \mathrm{C}$ for $30 \mathrm{sec}$, 
$54^{\circ} \mathrm{C}$ for $30 \mathrm{sec}$ and $72^{\circ} \mathrm{C}$ for $60 \mathrm{sec}$, and the final extension at $72^{\circ} \mathrm{C}$ for $5 \mathrm{~min}$.

Anchorage-independent growth assay. Each strain of logarithmic phase cells was trypsinized and dispersed to single cells, and each monoplast suspension was mixed with agarose in a final concentration of $0.35 \%$, respectively. Aliquots of $0.8 \mathrm{ml}$ containing 200 cells and $10 \%$ FBS were plated in triplicate on 24-well plates over a base layer of $0.6 \%$ agarose. Cells were incubated at $37^{\circ} \mathrm{C}$ in $95 \%$ air and $5 \% \mathrm{CO}_{2}$ for 14 days. Colonies $>75 \mu \mathrm{m}$ in diameter were counted under a microscope.

Cell-ECM adherence assay. The adhesion of cells to ECM was assayed as follows: 1 to 4 diluted Matrigel (BD Bioscience, San Jose, CA, USA) was coated at quantity of $25 \mu \mathrm{g} /$ well onto 24-well plates and left to dry at routine temperature in culture hood. Wells were washed 3 times with PBS and store at $4^{\circ} \mathrm{C}$ before use. Cells were collected and resuspended in serum-free RPMI-1640 medium containing $1 \%$ bovine serum albumin (Sigma). A total of $1.5 \times 10^{4}$ cells in $500 \mu 1$ were seeded into each substrate-coated well and plates were incubated for $20,40,60$ or $80 \mathrm{~min}$ at $37^{\circ} \mathrm{C}$ in $5 \% \mathrm{CO}_{2}$. Unattached cells were gently washed away with PBS. The attached cells were suspended and enumerated under microscopy.

Boyden chamber matrigel invasion and mobility assay. The invasive capacity of the cells was determined using a twocompartment Transwell Boyden chamber Matrigel invasion assay (Costar Corning Corporation, Burlington, MA, USA) as described by manipulator guide with slight modification. Briefly, the $8-\mu \mathrm{m}$-pore polycarbonate filters were coated with $120 \mu \mathrm{g}$ basement membrane Matrigel per filter (no Martigel needed for mobility assay), cells in $0.3 \mathrm{ml}$ of medium were added to each upper chamber and placed on top of a lower chamber prefilled with $0.6 \mathrm{ml}$ of serum-free culture supernatant of NIH3T3 cell lines, and then incubated at $37^{\circ} \mathrm{C}$ for $36 \mathrm{~h}$ (for mobility assay, incubation was for $8 \mathrm{~h}$ ). After incubation, the medium and/or gel was removed, and the polycarbonate filters with invaded cells were fixed in methyl alcohol at room temperature for $30 \mathrm{~min}$. They were then stained with Giemsa dye, washed, and mounted onto glass slides. The number of cells invaded was examined under a light microscope. Ten fields under a magnification of $\mathrm{x} 100$ for invasion or $\mathrm{x} 400$ for mobility assay were randomly selected, and the mean number of cells invading was calculated. Experiments were repeated in triplicate wells at least 3 times.

Cell wounding migrating assay. Cells were seeded in 6-well culture plates at a density of $2.5 \times 10^{5}$ cells per well. When cells were $\sim 80 \%$ confluent, scratch-wounds were created by scraping the confluent cell monolayer with a sterile pipette tip to make an $\sim 1.0 \mathrm{~mm}$ gap. After careful washing to remove detached cells, the cultures were incubated in serumfree medium with fibronectin (Sigma) at concentration of 16 $\mu \mathrm{g} / \mathrm{ml}$ for a further $48 \mathrm{~h}$. Wound healing was monitored at different time points with an inverted bright field microscope as described by Ateeq et al (16). The cell-free area was selected, measured, and quantified using the UTHSCSA ImageTool 3.0 software (an image processing and analysis programmed by researcher of the University of Texas Health Science Center at San Antonio) and calculated as percentage wound healing using the equation:

wound healing $(\%)=[1-($ wound area at Tx hours/wound area at T0)], where the Tx is the respective time point and T0 is the time immediately after wounding.

Intercellular calcium concentration analysis by fluo 3-Am staining. Intracellular $\mathrm{Ca}^{2+}\left(\left[\mathrm{Ca}^{2+}\right] \mathrm{i}\right)$ measurements were obtained with the fluorescent dyeing of $\mathrm{Ca}^{2+}$-sensitive dye fluo 3-Am (Amersham Pharmacia Bioscience, Piscataway, NJ, USA), then using a laser scanning confocal microscope with image acquisition and processing system (Radiance 2100, Bio-Rad Corporation). Fifty to sixty percent confluent cells on coverslips were washed with PBS 3 times and incubated with $10 \mu \mathrm{M}$ Fluo-3-Am (diluted from a $1.08 \mathrm{mM}$ stock in $20 \%$ pluronic acid-dimethylsulfoxide) for $30 \mathrm{~min}$ at $37^{\circ} \mathrm{C}$. After dye loading, cells were washed with PBS 3 times. Finally, $0.5 \mathrm{ml}$ PBS was reloaded in each dish and cells were used for analysis under a confocal microscope at the excited wavelength of $488 \mathrm{~nm}$. Five fields under a magnification of $\mathrm{x} 100$ were randomly selected, and the mean fluorescence density of $\sim 30$ cells was calculated for further statistical analysis.

Western blot analysis. Cells were rinsed twice with ice-cold PBS and scraped off, whole cell protein samples were prepared by lysing cells in a buffer composed of 1X PBS ( $\mathrm{pH} 7.4$ ), $1 \%$ ( $\mathrm{vol} / \mathrm{vol}$ ) nonidet $\mathrm{P} 40,0.5 \%$ sodium dexoycholate, $0.1 \%$ SDS, $1 \mathrm{mM}$ phenylmethylsulfonyl fluoride (PMSF), $20 \mu \mathrm{g} / \mathrm{ml}$ aprotinin, and $25 \mu \mathrm{g} / \mathrm{ml}$ leupeptin for $30 \mathrm{~min}$ at $4^{\circ} \mathrm{C}$. After the denaturation, equal amounts of the protein extracts were resolved by sodium dodecyl sulfate-polyacrylamide gel electrophoresis (SDS-PAGE) and transferred to a PVDF membrane. After blocking the membrane with buffer containing $20 \mathrm{mM}$ Tris- $\mathrm{HCl}(\mathrm{pH} 7.5), 140 \mathrm{mM} \mathrm{NaCl}$ and $5 \%$ nonfat milk for $2 \mathrm{~h}$ at room temperature, it was subsequently incubated with primary antibodies of anti-CD44, MMP2, P53, P16, VEGF (Santa Cruz Biotech) and MAG-2 for $\sim 1 \mathrm{~h}$ at room temperature, followed by washes and treatment with relevant horseradish peroxidase-labeled secondary antibody (Zhongshan Biotech, Beijing, P.R. China). These blots were developed using a chemiluminescent detection system (Santa Cruz Biotech). After stripping, the membrane was re-probed with an anti- $\beta$-actin antibody to control for equal protein loading and protein integrity.

Statistical analysis. All results were expressed as the means $\pm \mathrm{SD}$ (SE) of data obtained from three independent experiments performed in triplicate. Statistical significance was determined by the Student's t-test, general liner model process, ANOVA or Fisher's exact test of SPSS 13.0 software. The level of significance was set at $\mathrm{P}<0.05$.

\section{Results}

Physiological location of MAG-2 protein intercellular and expression profile of the gene in human multi-tissues. The 
A

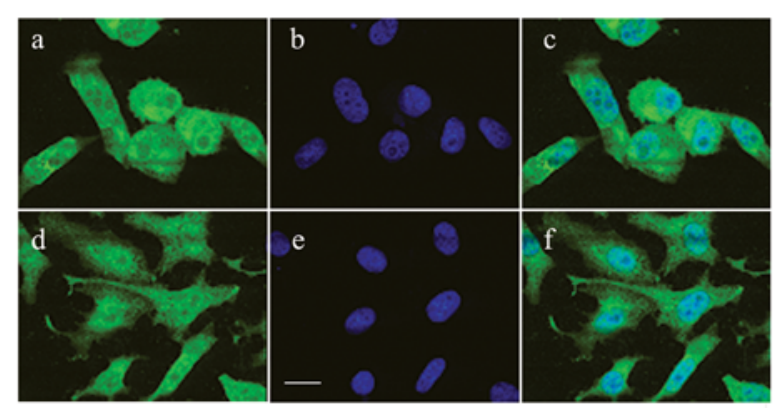

B

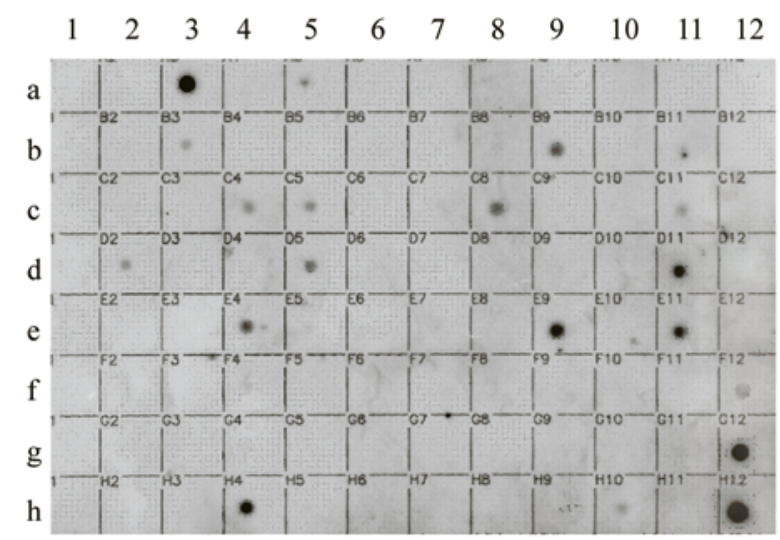

a1 whole brain
a2 cerebellum, left
a3 Substantia nigra
a4 heart
a5 esophagus
a6 colon,transvers
a7 kidney
a8 lung
a9 liver
a10 leukemia,HL-60
a11 fetal brain
a12 Yeast total RNA
b1 cerebral cortex
b2 cerebellum, right
b3 Accumbens nucleus
b4 aorta
b5 stomach
b6 colon,,desending
b7 Skeletal,muscle
b8 placenta
b9 pancreas
b10 Hela,S3
b11 Fetal,heart
b12 Yeast,tRNA
c1 frontal lobe
c2 corpus callosum
c3 thalamus
c4 atrium,left
c5 duodenum
c6 rectum
c7 spleen
c8 bladder
c9 Adrenal,gland
c10 leukemia,K562
c11 Fetal,kidney
c12 E.coli,rRNA
d1 parietal lobe
d2 amygdala
d3 Pituitary,gland
d4 atrium,right
d5 jejunum
d7 thymus

d8 uterus

d9 Thyroid,gland

d10 leukemia, MOLT-4

d11 Fetal,liver

d12 E.coli,DNA

el occipital lobe

e2 Caudate,nucles

e3 Spinal,cord

e4 ventricle,left

e5 ileum

e7 Peripheral blood leukocyte

e8 prostate

e9 Salivary,gland

e10 Burkitt's, lymphoma'Raji

e11 Fetal,spleen

e12 Polyr(A)

fl temporal lobe

f2 hippo-campus

f4 ventricle,right

f5 ilocecum

f7 Lymph,node

f8 testis

f9 Mammary,gland

f10 Burkitt's, lymphoma'Daudi

f11 Fetal,thymus

f12 Human,C0t-1 DNA

g1 P.G, cerebral cortex

g2 Medulla,oblongata

g4 inter-ventricular septum

g5 appendix

g8 ovary

g10 Colorectal adeno

carcinoma,SW480

g11 Fetal,lung

g12 Human DNA100ng

h1 pons

h2 putamen

h4 apex of the heart

h5 colon, ascending

h7 trachea

h10 A549

h12 human DNA500ng

Figure 1. MAG-2 cellular location and the gene expression profile analysis. (A) Physiological location of MAG-2 protein intercellularly. PLA801D and HepG2 cells on coverslips were fixed in $4 \%$ paraformaldehyde then incubated with diluted anti-MAG- 2 polyclone antibody at $4{ }^{\circ} \mathrm{C}$ overnight, followed by FITC-linked second antibody and 4',6-diamidino-2-phenylindole (DAPI) staining, and observed under a confocal microscope. MAG-2 was demonstrated to present diffusely in the cytoplasm and nucleus in both PLA801D (a, fluorescence of FITC; b, DAPI; c, merged) and HepG2 (d, fluorescence of FITC; e, DAPI; f, merged) cells (bar $=10 \mu \mathrm{m}$ ). (B) MAG-2 gene expression profile in multiple adult and fetal tissues. Commercially available human multiple tissue expression array was hybridized with radioactive probe synthesized as per the MAG-2 cDNA template by Klenow DNA polymerase fragment. MAG-2 mRNA was found expressed in the nigra, salivary gland, cardiac apex, left ventricle, pancreatic gland, bladder, jejunum and amygdale tissue of adult, and liver, spleen, heart and kidney of fetus.

endogenous MAG-2 protein in PLA801D cells were visualized by immunofluorescence staining under laser scanning confocal microscope, as shown in Fig. 1Aa-c, the granulated protein of MAG-2 was seen dispersed in the entire cell including cytoplasm and nucleoplasm, but MAG-2 was not detected in the culture supernatant of either PLA801C or PLA801D by Western blotting (data not show), suggested a non-secreted character of the protein. To exclude it being an artificial phenomenon, we repeated the fluorescence dyeing using HepG2 cells and obtaines similar results as in PLA801D (Fig. 1 Ad-f). In MTE dot hybridization analysis, MAG-2 mRNA was found expressed maximally in the nigra, salivary gland and at a lower level in the cardiac apex, left ventricle, pancreatic gland, bladder, jejunum and amygdale of adult tissue (Fig. 1B, lanes 1-9). In fetal tissues (Fig. 1B, lane 11), the messenger for MAG-2 was found in the liver, spleen and at a much lower level in heart and kidney but not in the brain, lung and thymus. Specificity of the probe was confirmed by the absence of signal in the control samples (Fig. 1B, lane 12).

Effects of MAG-2 on cells anchorage-independent growth, adherence, mobility and invasion. To verify the MAG-2 effects on metastasis-related behavior in vitro, a MAG-2 hyperexpression PLA801C strain (termed as CMAG2+) and a hypoexpression PLA801D (termed as DMAG2-) were established by transfecting recombinant plasmids pcDNA3.0/MAG2S and pcDNA3.0/MAG2AS to PLA801C and D, respectively. The exogenous enhancement and inhibition of MAG-2 expression in transfected cell strains were confirmed by RTPCR (Fig. 2A). Densitometric analysis of the bands indicated that mRNA level of MAG-2 in CMAG2+ was 7 times more than that of Cvector, whereas in DMAG2-, anti-sense plasmid reduced the MAG-2 mRNA level by $60 \%$. Cell strains 
A

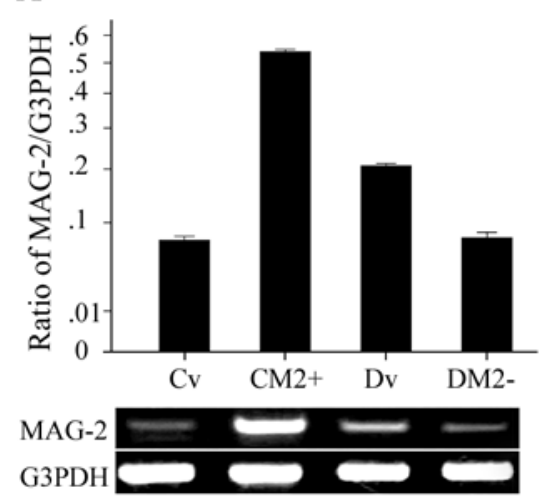

D

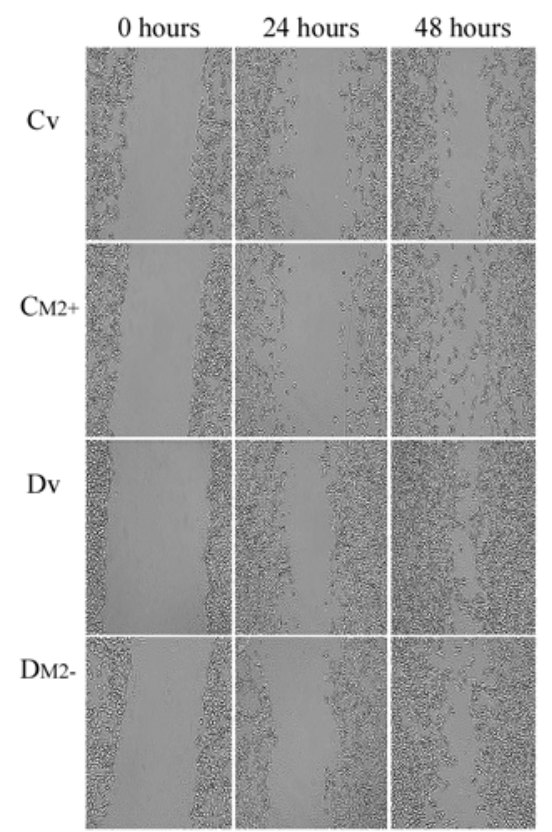

B

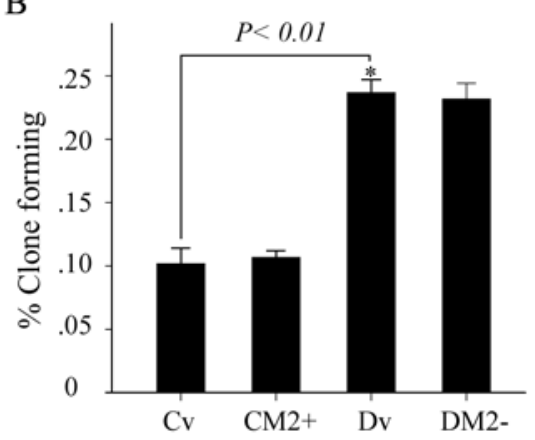

E

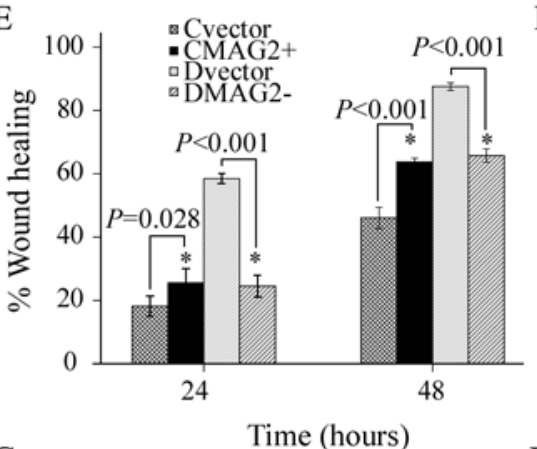

G

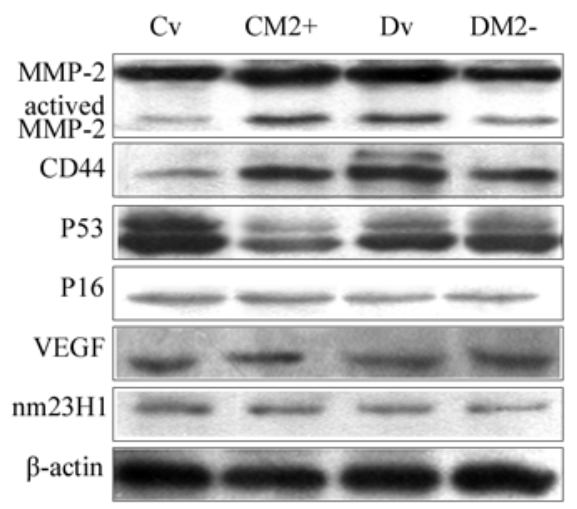

C

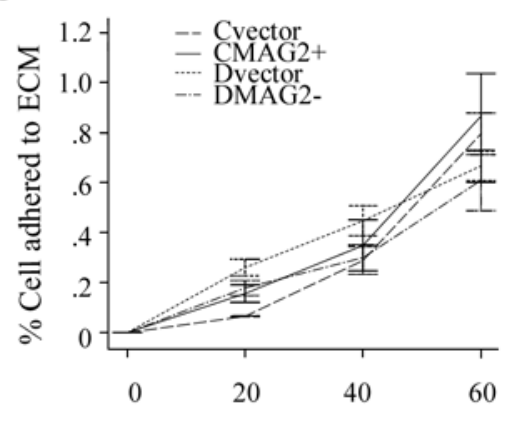

Time (minutes)

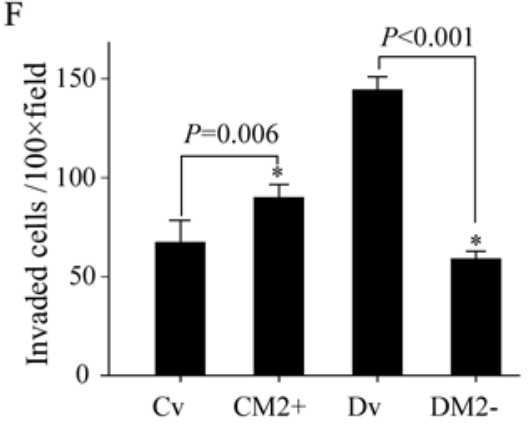

$\mathrm{H}$

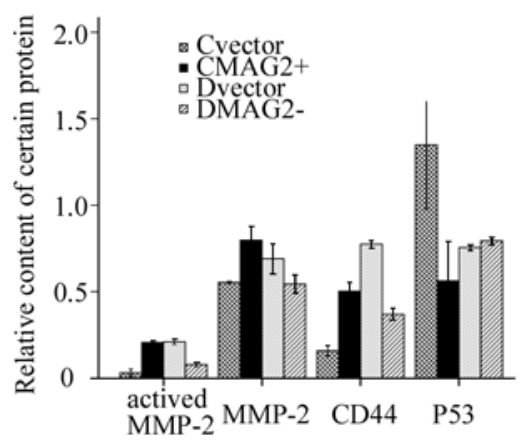

Figure 2. Effects of MAG-2 on the anchorage-independent growth capability, ECM adherence, mobility, invasion and proteins concentration related to tumor metastasis. PLA801C and D cells were transfected with sense and anti-sense MAG-2 vectors respectively, as show in (A), mRNA level of MAG-2 in CMAG2+ had elevated 7 times, whereas in DMAG2-, MAG-2 mRNA was depressed by anti-sense vector by $60 \%$. Then metastatsis-related characteristics of the four stable transfected cell strains, Cvector (Cv), CMAG2+ (CM2+), Dvector (Dv) and DMAG2- (DM2-) were evaluated. Anchorage-independent growth capability was determined by clonogenetic assay in soft agar as described in the Materials and methods. The data were analyzed by ANOVA process of SPSS, as shown in (B), no significant difference between CMAG2+, DMAG2- and their controls was observed, but notable difference on clone forming capability existed between the strains D and C (Cvector vs. Dvector: F=147.7, $\mathrm{P}<0.01)$. (C) The results of cell-EMC adherence assay. Significant differences of CMAG2+ vs. Cvector ( $\mathrm{F}=4.847, \mathrm{P}=0.049)$ and $\mathrm{DMAG} 2$ - vs. Dvector $(\mathrm{F}=4.847, \mathrm{P}=0.017)$ were verified by using the repeated measures process of SPSS13.0 statistical software. Lines are drawn between points only for the sake of clarity, as the adherence ability association between time points is not linear. Results of wound healing migration assay are depicted in panels (D) and (E). Cells were seeded at the same density and grown as monolayer and wounded by sterilized tip. \%Wound healing was recorded at different time points, and percentage of wound healing with respect to T0 was calculated using the described equation. The invasion analysis was done using Matrigel-coated invasion chamber assays with $1 \times 10^{5}$ model cells per 24 -well plate. After data being analyzed by method of Student's t-test, significant differences between each paired test-control cells were drawn, as showed in panel (F). Panels (G) depicts MAG-2 effects on intracellular level of certain proteins analyzed by Western blotting. MMP-2, CD44 and especially the active MMP-2 have positive correlation with MAG-2 gene expression level, as shown in (H). All data presented in panels (B) to (F) are expressed as mean \pm SD (SE), from three independent experiments performed in triplicate, in panels $(\mathrm{A})$ and $(\mathrm{H})$ error bar stand for SD of each sample was measured in triplicate. Significant difference from the control is represented by asterisk (relevant $\mathrm{P}$-values are marked on each panel).

CMAG2+, DMAG2- and each vector control (named as Cvector and Dvector) were used as the model cell for the cytological experiments.

Anchorage-independent growth capability of CMAG2+, DMAG2- and their vector control were determined by clonegenetic assay in soft agar. After 14 days of culture in an incubator, clones $>75 \mu \mathrm{m}$ in diameter were enumerated under a microscope. Clone forming rate for Cvector, CMAG2+, Dvector and DMAG2- were $(10.17 \pm 1.25) \%,(10.67 \pm 0.58) \%$, $(23.67 \pm 1.04) \%$ and $(23.17 \pm 1.52) \%$, respectively. Statistical results show that the variance between colonization of CMAG2+, DMAG2- and their controls had not reached the 
level of significance, but significant differences existed between strain C and D (Cvector vs. Dvector: $F=147.7$, $\mathrm{P}<0.01$ ) (Fig. 2B).

Interaction between cell and ECM is the fatal aspect of all the steps included in the metastatic process, adhesive capacity changing in cell-cell and cell-ECM in the tumor nests are the initiation of invasion (17). Results from our cell-ECM adherence assay studies show that MAG-2 had adherence promoting function in lung cancer cells (Fig. 2C). At the time points of 20,40 and $60 \mathrm{~min}$, the amount of attached cells observed in PLA801C cells transfected with pcDNA3.0/MAG2S plasmid, expressed as a percentage of total seeded cells, were $13.5,30.4$ and $75.3 \%$, compared with $5.7,25.1$ and $69.2 \%$ in control cells; whereas the adherence rates of PLA $801 \mathrm{D}$ cells transfected with pcDNA3.0/ MAG2AS, 15.6, 26.1 and 53.0\% at three succeeding time points, was reduced compared with $22.6,39.1$ and $58.1 \%$ of vector control cells. Significant differences of CMAG2+ vs. Cvector $(\mathrm{F}=4.847, \mathrm{P}=0.049)$ and DMAG2- vs. Dvector $(\mathrm{F}=4.847, \mathrm{P}=0.017)$ were verified by using the repeated measures process of SPSS13.0 statistical software.

The effect of MAG-2 on cell migration was analyzed using wound healing tests. A significant (vs. Cvector: $\mathrm{F}=136.1$, $\mathrm{P}<0.001)$ increase in wound healing $(\%)$ was observed in MAG-2 hyper-expressional CMAG2+ cells comparing with control cells at 24 and $48 \mathrm{~h}$ after wounding. At the same time, healing ability of DMAG2- cells, whose MAG-2 expression was partly blocked by anti-sense mRNA, were found much lower (vs. Dvector: $\mathrm{F}=136.1, \mathrm{P}<0.001$ ) than vector control cells (Fig. 2D and E).

MAG-2 was also proven to be a stimulative of lung cancer cell invasion in our studies. The invasion analysis was done using Matrigel-coated invasion chamber assays with CMAG2+, DMAG2- and their vector control cells $\left(1 \times 10^{5}\right.$ cells per well of 24-well plate). We found that excessive expression of extra-generational MAG-2 in PLA801C cells would lead to a more aggressive $\mathrm{C}$ strain. Accordingly, invasion of PLA801D cells was restrained if the gene MAG-2 mRNA level dropped. Statistical evaluation of the data, comparing the number of penetrating cells of each tested cell type with its control, using Student's t-test, showed that the difference on invasion property was significant at $\mathrm{P}=0.006$ between Cvector and CMAG2+, and at $\mathrm{P}<0.001$ between Dvector and DMAG2- cells (Fig. 2F).

Effects of MAG-2 on proteins involved in invasion and metastasis. In order to investigate the mechanism by which MAG-2 stimulates cell invasion, we tested certain proteins, which was previously reported to be involved in cell invasion and metastasis, including MMP-2, CD44 and nm23H1 by Western blot assay (Fig. 2G and H). Bands densitometric analysis indicated that excessive expression of MAG-2 leads to a significant elevation in the level of MMP-2 ( 1.6 times vs. control), especially the activated MMP-2 ( $\sim 10.9$ times vs. control). We also found that CD44 level in CMAG2+ was 3.3 times higher than that of control cells, which strongly suggested that MAG-2 has the function of cell-ECM adherence promoting by elevating CD44 concentration. In accordance with the results from PLA801C strain, we observed that MMP-2 and CD44 were markedly depressed in DMAG2-
A
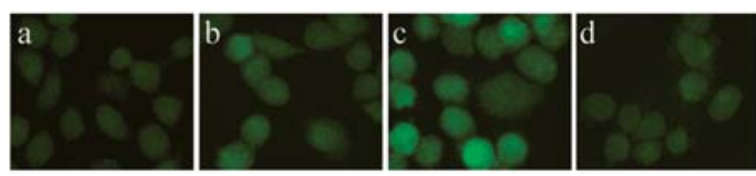

B

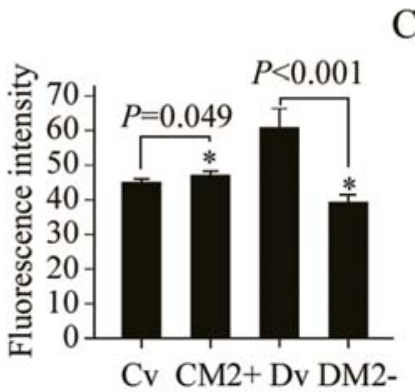

$\mathrm{C}$

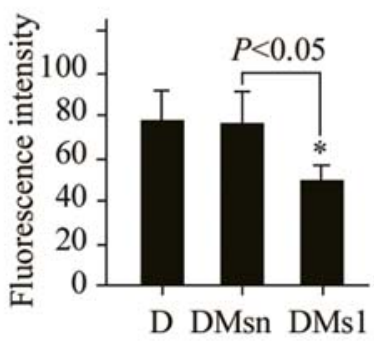

Figure 3. Effects of MAG-2 on intracellular free calcium concentration. (A) Representative fluorescence images of Cvector (a), CMAG2+ (b), Dvector (c) and DMAG2- (d) cells under confocal microscope after staining by Fluo-3 Am. (B) and (C) show the fluorescence intensity of cells from each type of cell strain. Bar, SE of fluorescence intensity of cells from five random fields ( $\sim 30$ cells per field). Cv, Cvector; CM2+, CMAG2+; Dv, Dvector; DM2-, DMAG2-; D, PLA801D. Significant difference from the control is represented by asterisk (relevant P-values are marked on each panel).

cells caused by partial inhibition of the MAG-2 expression with anti-sense mRNA in PLA801D cell strain. We found that the variation of MAG-2 mRNA had no effect on VEGF, $\mathrm{P} 16$ and $\mathrm{nm} 23 \mathrm{H} 1$. Moreover, protein $\mathrm{p} 53$ was proven to be reduced in $\mathrm{CMAG} 2+$ but no corresponding elevation was detected in DMAG2-, which might be due to the difference on chromosome 17 (on which the p53 gene located) between PLA801D and C strains (12).

Effects of MAG-2 on intracellular free calcium concentration. Previous in vitro and in vivo studies have suggested that calcium signalling is involved in virtually all cellular processes of cancer cells, including invasion and metastasis $(18,19)$. To validate the influence brought by MAG-2 on calcium variation in cells, we measured the concentration of free intracellular calcium $\left(\left[\mathrm{Ca}^{2+}\right] \mathrm{i}\right)$ in the cells by the methods of fluo 3 -Am fluorescent probe staining (Fig. 3A). Results of the analysis showed that overexpression MAG-2 stimulated a significant increase of $\left[\mathrm{Ca}^{2+}\right] \mathrm{i}$ in PLA801C cells $(\mathrm{t}=2.794, \mathrm{P}=0.049)$, whereas in the DMAG2- cells, reduced MAG-2 transcripts led to a lower $\left[\mathrm{Ca}^{2+}\right] \mathrm{i}$ level than in the Dvector cells $(\mathrm{t}=7.919$ $\mathrm{P}<0.001)$ (Fig. 3B). Changes in the cytosolic-free $\mathrm{Ca}^{2+}$ concentration may be due either to release from the intracellular $\mathrm{Ca}^{2+}$ stores or to influx from the extracellular medium, through the opening of plasma membrane calciumpermeable channels.

Altering of mobility, invasion, adherence characteristics and $\left[\mathrm{Ca}^{2+}\right] \mathrm{i}$ in PLA801D cells with MAG-2 knockdown by RNA interference. Previous studies have stated (20-22) that RNA interference, which produced double-stranded RNA (dsRNA) $<30$ nucleotides intracellular, is a more appropriate technique than full-length or big fragment antisense mRNA introducing when silencing a certain gene. In order to exclude the 
A

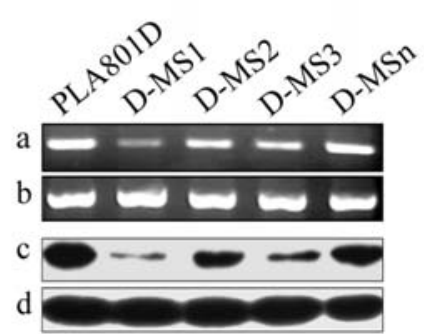

B

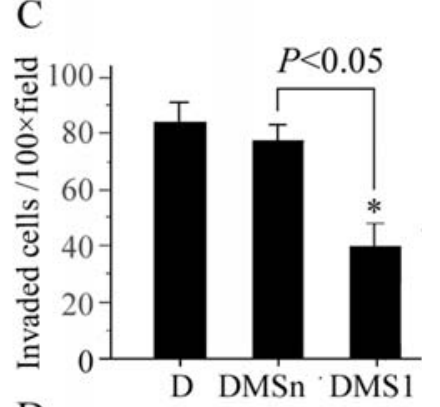

$\mathrm{D}$
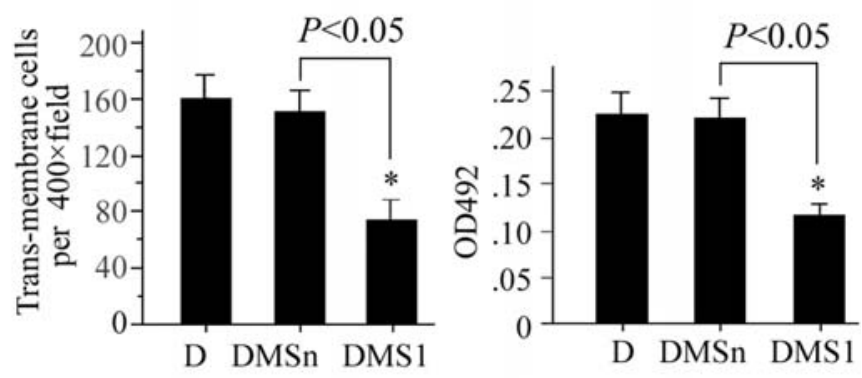

Figure 4. Influence of MAG-2 on migration, invasion and ECM adherence capability of PLA801D cells, confirmed by RNAi. Three complementary strands of oligonucleotides, MS1, MS2 and MS3 specifically targeting MAG-2 had distinct effects on MAG-2 gene silencing, as shown in panel (A), MS1 is the most efficient among all sequences, which was proven in both mRNA ( $a$ and $b$ : RT-PCR results of MAG-2 and intra control GAPDH) and protein level (c and d: Western blot results of MAG-2 and B-actin control). The functional experiments also demonstrated that the MAG-2 gene knock-down would lead to a significant depression on cell mobility (B), invasion (C) and adherence to ECM (D) in vitro. All data presented are expressed as mean $\pm \mathrm{SD}$, from three independent experiments performed in triplicate. Significant difference from the control is represented by asterisk (relevant P-values are marked on each panel).

possible side effects caused by dsRNA (longer than 80 nucleotides) through PKR (RNA-dependent protein kinase) activation, RNA interference technique was employed to knock-down the MAG-2 genes in PLA801D cells. Three oligonucleotides, MS1, MS2 and MS3 were synthesized and cloned into the multiple cloning sites of pSilencer 2.1-U6, then reconstructed vectors were introduced into PLA801D cells, respectively. mRNA and protein level of MAG-2 were assayed in cell strains, D-MS1, D-MS2, D-MS3 (termed corresponding to different oligonucleotides introduced), D-MSn (vector control) and PLA801D. As shown in Fig. 4A, MS1 was the most efficient silencer of MAG-2 gene in all three sequences.

Thereafter, the mobility and invasive properties of D-MS1 were analyzed in vitro using the methods of Transwell system. Results demonstrated that the capabilities of D-MS1 cells on mobility and invasion were significant reduced by $50.7 \%$ and $48.3 \%$, respectively, comparing with that of vector control cells

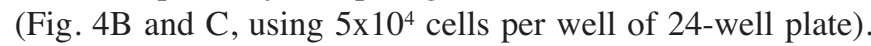
Adherence ability of D-MS1 cells was also tested by the method described in Kumagai et al (23). Cells (seeded in 96well culture plate at an concentration of $2.0 \times 10^{4}$ cells/well) were incubated at $37^{\circ} \mathrm{C}$ for $1 \mathrm{~h}$ and stained by $3-(4,5)$ dimethylthiahiazo (-z-y1)-3, 5-di-phenyltetrazolium bromide (MTT) after nonattached cells were removed by washing with PBS. As shown in Fig. 4D, the absorbency value of
A

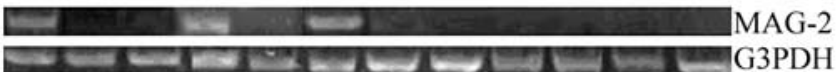

B

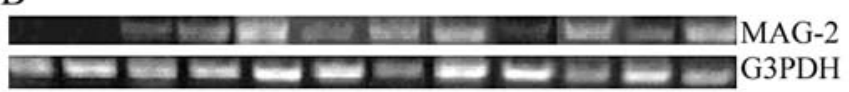

Figure 5. MAG-2 mRNA detection in tumor tissues with or without overt metastasis. Detection rate of MAG-2 mRNA in tumor tissue from nonmetastatic patients was much lower $(3 / 12$, as shown in $A)$ than the rate in tissues from patients with overt metastatic lesion (10/12, as shown in panel B).

D-MS1 cells was depressed significantly by $46.9 \%$ comparing with D-MSn cells $(\mathrm{P}<0.05)$, which suggested MAG-2 is capabable of adhesion stimulation.

Intracellular-free calcium concentration in D-MS1 cells was determined likewise to confirm the effects of MAG-2 on calcium flux and exchanges. In agreement with the results from anti-sense expression vector transfected DMAG2-cells, we also observed that $\left[\mathrm{Ca}^{2+}\right] \mathrm{i}$ was markedly decreased in D-MS1 cells in the experiments and the statistical analysis demonstrated a significant difference $(\mathrm{P}<0.05)$ between $\mathrm{D}$ MS1 and D-MSn (Fig. 3C).

Expression of MAG-2 in tumor tissue from lung cancer patients with or without pathologically proven metastasis. To verify the correlation between MAG-2 transcription and metastatic situation of lung cancer clinically, we then measured mRNA levels of MAG-2 in tumor tissues from 24 lung cancer patients using RT-PCR assay. All patients' diseases were confirmed by pathological diagnosis. Among those patients, twelve had metastatic lesions in distance organs intra- or post-operation, whereas the others did not. The RT-PCR analysis was able to distinguish between patients with metastatic foci and those without for MAG-2 mRNA expression in cancer tissues of 10/12 versus 3/12, $\mathrm{P}=0.006$, by Fisher's Exact test (Fig. 5).

\section{Discussion}

Although research on transcriptome (17) and interaction between cancer cells and host normal cells (24) have uncovered new, unexpected insights into the mechanism of metastasis, traditional studies, which had identified several required steps and a rational list of genes (and proteins) mediating each, are still one of vital aspects on exploring this last and lethal step in tumor progression as per the theory of 'seed and soil' (6). MAG-2 was initially selected as the homological full-length cDNA of a fragment of SSH library, which was constructed using two sister giant-cell lung carcinoma cell strains with different metastasis potential in our laboratory, and MAG-2 was proved to have a much higher mRNA level in highly metastatic lung cancer cell strains than poorly metastatic cells by Northern blotting $(9,13)$.

The MAG-2 gene has a length of 5.2-k base pairs, locates on chromosome 2q35 and is well homologous to FAM134A (family with sequence similarity 134, member A) in GenBank; its 2.4-kbp cDNA sequence is assembled by 9 exons (Fig. 6A). Complete cDNA sequence revealed a single open reading 
A

Chromosome: 2; Location: 2q35

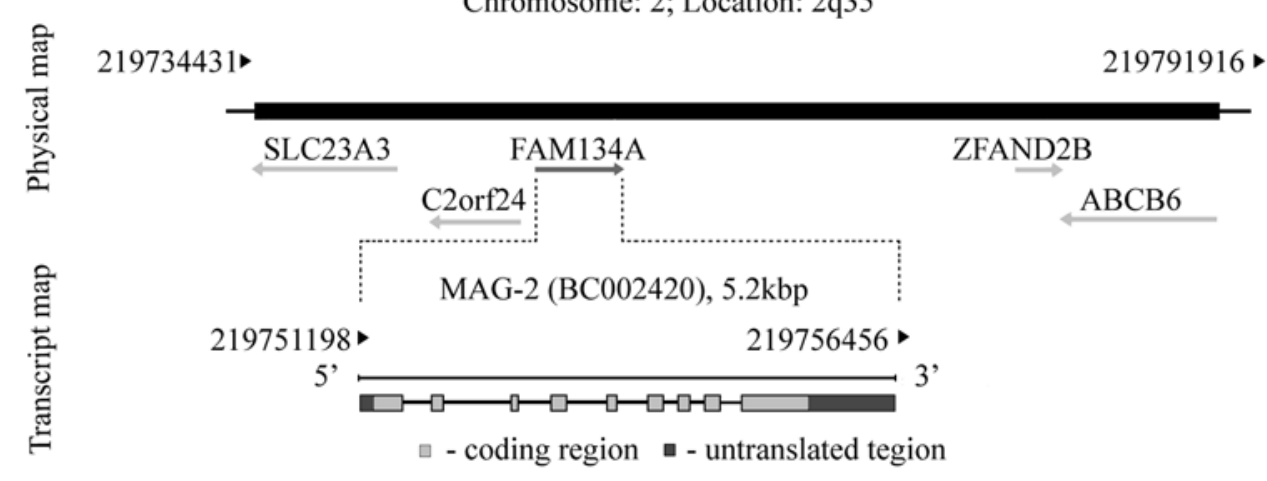

B

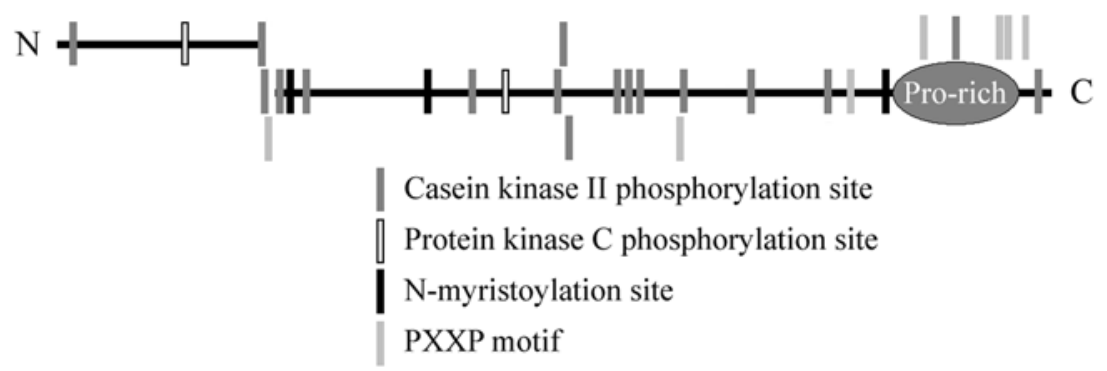

Casein kinase II phosphorylation sites:

6-9: SeaE, 100-103: SspE, 101-104: SpeE, 107-110: SdsE, 122-125: SvpE, 203-206: TrlE, 248-251: TesE, 250-253: SesE, 252-255: SeaE, 275-278: TdsE, 280-283: SdeE, 285-288: SilE, 316-319: SepE, 354-357: SeeE, 386-389: SpqD, 457-460: TteD, 502-505: SdqE

Protein kinase $\mathrm{C}$ phosphorylation sites:

69 - 71: SIR, 215 - 217: SmK

$\mathrm{N}$-myristoylation sites:

111-116: GAgsGA, 175-180: GImiSY, 409-414: GGltAL

PxxP motif:

102-105: PeeP, 315-318: PseP, 395-398: PggP, 437-440: PpnP, 480-483: PetP, 484-487: PkpP, 491-494: PlgP

Proline-rich region profile :

415 - 494: pgtlspplclvgsdpapspsilppvpqdspqplpapeeealttedfelldqgeleqlnaelglepetppkppdapplgp

Figure 6. Identification of the MAG-2 gene. (A) Physical and transcript map of the MAG-2 gene region. Physical map shows a 57.5-kb segment of human chromosome 2q35. The region include five validated or predicated protein-coding genes: SLC23A3 (solute carrier family 23 - a family of nucleobase transporters - member 3, validated), C2orf24 (predicted), ZFAND2B (zinc finger, AN1-type domain 2B gene, provisional), ABCB6 (ATP-binding cassette, sub-family B member 6, reviewed) and FAM134A (MAG-2). The transcript map of MAG-2 shows nine exons that span 5.2-kb of genomic DNA. The ORF is present in all exons. (B) The deduced polypeptides of MAG-2 gene. The pattern shows all the predicted profiles and its relative positions on the hypothetical proteins composed of 512 amino acids, including possible 22 modified sites, 7 PXXP motifs and 1 praline-rich region.

frame (ORF) of 1539 nucleotides encoding a protein of 512 amino acids with an approximate molecular mass of $55 \mathrm{kDa}$, which was proved to be true in Western blotting (15). A Proline-rich region in C-terminal, seven repeats of sequence PXXP and tens of possible phosphorylation or N-myristoylation sites were found after scanning the protein sequence by web-based software of protein domains, families and functional site finder: ScanProsite (available from URL: http://www. expasy.org/prosite/) and ProfileScan (available from URL: http://hits.isb-sib.ch/cgi-bin/motif_scan) (Fig. 6B). In RNA blot assay, we observed MAG-2 mRNA transcription in many kinds of adult and fetal tissues, and we also found that MAG-2 protein has pan-cellular location in cancer cells by immunofluorescence. Therefore, MAG-2 was proven to be a genuine gene with derived mRNA and peptide sequence rather than a hypothetical gene predicted by bioinformatics.

We demonstrated that MAG-2 plays important roles in promoting tumor metastasis in our experimental model: overexpression of MAG-2 leads to an enhancement of migration, invasion and adherence to ECM, and reduction of MAG-2 leads to suppression of those capabilities. We also proved in our study that MAG-2 could elevate the protein level of CD44 
and MMP-2 intracellularly, which could be one of the molecular mechanisms underlaying the invasion, mobility and ECM adherence promoting characteristics of MAG-2. As a transmembrane glycoprotein, CD44 was found in a wide variety of tissues and its known functions, including cellular adhesion (aggregation and migration), hyaluronate degradation, lymphocyte activation, lymph node homing, myelopoiesis and lymphopoiesis, angiogenesis, and release of cytokines, were well reviewed by Sneath and Mangham (25).

Increased expression of CD44 can enhance binding to hyaluronate and a pericellular matrix of hyaluronate might decrease the affinity of a cell to surrounding hyaluronate deficient cells by interfering with adhesion processes, thus leading to detachment; the first step of metastasis. Since linked to the cell's cytoskeleton (26), CD44 could initiate a series of biochemical activities intracellularly after binding to hyaluronic acid (HA), finally the CD44-HA binding would lead to enhancement of cell mobility characteristics $(27,28)$, and the property of CD44 to degrade hyaluronate after taking up the ligand could allow tumor cells to escape entrapment within hyaluronate-rich environments, thus cells could move from one place to another by this CD44-HA binding and degrading circulation (29). Furthermore, CD44 was also found to have positive interaction with MMP-2, a fully documented migration facilitating molecule, in many types of lung cancer cell lines (30-32), thus, CD44 and MMP-2 have mutual promoting effects on cell migration, these effects might also exist in our model of cell line PLA801 and be the reason for MAG-2 function.

As previously discussed, MAG-2 contains a proline-rich domain with abundant proline residues $(26 \%)$, a feature found in many proteins that play an indispensable role in mediating a multitude of protein-protein interactions (33). MAG-2 also contains 7 repeats of the sequence PXXP. This motif has been shown to play a role in signal transduction by interacting with SH3 domain containing proteins (34), and the presence of this motif in MAG-2 suggests that the protein might function as a binding protein of $\mathrm{SH} 3$ domain-containing proteins and could be involved in regulation or modification of these binding partners, many of which play important roles in signal transduction pathways (35). The two types of structure, proline-rich sequences and PXXP motif might be the molecular basis of the effect on CD44 and MMP-2 brought by MAG- 2 directly or indirectly.

We verified that intracellular-free calcium concentration had the same vibrating trend with MAG-2 mRNA level, which suggested that MAG-2 might have influence on the intracellular calcium. Calcium ions are involved in virtually all cellular processes spatially and temporally; among others, they control cell survival, proliferation and apoptosis, regulate a plethora of intracellular enzymes located in the cytoplasm, nucleus and organelles, and influence cell polarization and locomotion $(19,36)$. Some research has demonstrated that highly metastatic tumor cells have a much higher free intracellular calcium concentration and lower regulating capability on $\left[\mathrm{Ca}^{2+}\right] \mathrm{i}$ than poorly metastatic tumor cells, as concluded in our experiments. These data suggested that distinct calcium ion regulatory mechanisms were present in poorly and highly metastatic cells (37-39).
The differences on regulation of certain low voltageactivated and nonvoltage-gated channels on the cell periphery might be one of the reasons for such diversity of calcium level between cells with different metastatic potential (19). $\mathrm{Ca}^{2+}$ wave, which was caused by rapid influx of calcium extracellular through the two types of channels and which travel on the periphery of cytoplasm, more exactly, the biochemical effects on intermediate filaments brought by $\mathrm{Ca}^{2+}$ wave might be the mechanisms in cell migration regulated by calcium $(19,38)$. Moreover, FAM134A proteins are transmembrane or integral proteins to membrane (http:// www.ncbi.nlm.nih.gov) and have interactions with KIAA1377, a protein with protein-binding function $(40,41)$. Consequently, interaction directly or indirectly with component of ion channel of calcium could be a reason for intracellular calcium concentration increase caused by MAG-2. Therefore, we hypothesized that MAG-2 could elevate $\mathrm{Ca}^{2+}$ ion concentration in model cells through proteinprotein interaction and that might be another approach besides interaction with CD44 and MMP-2 by which MAG-2 demonstrate its migration-promoting function, but the detailed aspects need further research.

In addition to cytological experiments, we also found that MAG-2 mRNA has a significantly high positive rate in the tumor tissues from lung cancer patients with diagnosed metastasis comparing with the rate in tissues from non-metastatic patients by RT-PCR. All the little tissue blocks of lung cancer used for total RNA extraction were selected carefully by pathologist, to make sure that the results of RT-PCR was bona fide reflections of MAG-2 transcription in tissues of different metastatic state. The results from tissue are more reliable due to less artificial disturbance. Therefore, the clinical results could give support to such a conclusion that the expression of MAG-2 gene has a positive correlation with metastasis characteristics of lung cancer. MAG-2 was identified as a metastasis promoting gene in our laboratory, but the detailed mechanism by which it is involved in the metastasis process is still undefined, further study is needed to search for the molecules which have direct interaction with MAG-2 protein and address the potential biochemical function of MAG-2 in lung cancer cells.

\section{Acknowledgements}

This study was supported by grants from the National Natural Science Foundation of China (to Yinglin Lu, No. 30470389) and The Major State Basic Research Development Program of China (to Yinglin Lu, No. 2002CB513105). We thank Sample Bank of Beijing Cancer Hospital for providing all the lung cancer tissues.

\section{References}

1. Sahai E: Illuminating the metastatic process. Nat Rev Cancer 7: 737-749, 2007.

2. Yang J: Exploring the molecular basis of tumor metastasis by microarray analysis. Assay Drug Dev Technol 4: 483-488, 2006.

3. Pedraza-Farina LG: Mechanisms of oncogenic cooperation in cancer initiation and metastasis. Yale J Biol Med 79: 95-103, 2006.

4. Bernards R and Weinberg RA: A progression puzzle. Nature 418: 823, 2002 . 
5. Padua D, Zhang XH, Wang Q, Nadal C, Gerald WL, Gomis RR and Massague J: TGFbeta primes breast tumors for lung metastasis seeding through angiopoietin-like 4. Cell 133: 66-77, 2008.

6. Fidler IJ: The pathogenesis of cancer metastasis: the 'seed and soil' hypothesis revisited. Nat Rev Cancer 3: 453-458, 2003.

7. Jonsson JJ and Weissman SM: From mutation mapping to phenotype cloning. Proc Natl Acad Sci USA 92: 83-85, 1995.

8. Diatchenko L, Lau YF, Campbell AP, Chenchik A, Moqadam F, Huang B, Lukyanov S, Lukyanov K, Gurskaya N, Sverdlov ED and Siebert PD: Suppression subtractive hybridization: a method for generating differentially regulated or tissue-specific cDNA probes and libraries. Proc Natl Acad Sci USA 93: 6025-6030, 1996.

9. Zhang J, Wang Y, Wang T and Lu Y: Differentially expression genes in human giant-cell lung cancer lines with different metastatic potentials. Chin J Oncol 26: 590-593, 2004.

10. Jiang D, Ying W, Lu Y, Wan J, Zhai Y, Liu W, Zhu Y, Qiu Z, Qian $X$ and He F: Identification of metastasis-associated proteins by proteomic analysis and functional exploration of interleukin18 in metastasis. Proteomics 3: 724-737, 2003.

11. Lu Y, Huang $\mathrm{J}$ and $\mathrm{Li} \mathrm{X}$ : Influence of immune deficient mice to the metastatic phenotypes of the heterogenic clonal humanlung cell carcinoma sublines. Chin J Oncol 11: 3-7, 1989.

12. Mei L, Lezhen C, Po Z, Zheng G, Yali L and Nan L: Study on the metastatic mechanisms of human giant-cell lung carcinoma comparison between the strains C and D. Asian Pac J Allergy Immunol 16: 167-176, 1998.

13. Zhang J, Meng Y, Du Z, Chen Z, Ling X, Xu Y and Lu Y: Identification of new metastasis associated genes MAG-1 and MAG-2. Chin J Lung Cancer 6: 460-463, 2003.

14. Wang Y, Lu C, Wei H, Wang N, Chen X, Zhang L, Zhai Y, Zhu Y, Lu Y and He F: Hepatopoietin interacts directly with COP9 signalosome and regulates AP-1 activity. FEBS Lett 572: 85-91, 2004.

15. Liu G, Xu Y, Jiang Y, Jia H, Pan H, Du Z and Lu Y: Polyclonal antibody specific to human tumor metastasis associated mag-2: preparation and identification. Bull Acad Military Med Sci 31 231-233, 2007.

16. Ateeq B, Unterberger A, Szyf M and Rabbani SA: Pharmacological inhibition of DNA methylation induces proinvasive and prometastatic genes in vitro and in vivo. Neoplasia 10: 266-278, 2008.

17. Steeg PS: Tumor metastasis: mechanistic insights and clinical challenges. Nat Med 12: 895-904, 2006.

18. Capiod T, Shuba Y, Skryma R and Prevarskaya N: Calcium signalling and cancer cell growth. Subcell Biochem 45: 405-427, 2007.

19. Huang JB, Kindzelskii AL, Clark AJ and Petty HR: Identification of channels promoting calcium spikes and waves in HT1080 tumor cells: their apparent roles in cell motility and invasion. Cancer Res 64: 2482-2489, 2004

20. Medema RH: Optimizing RNA interference for application in mammalian cells. Biochem J 380: 593-603, 2004.

21. Williams BR: Role of the double-stranded RNA-activated protein kinase (PKR) in cell regulation. Biochem Soc Trans 25: 509-513, 1997.

22. Caplen NJ, Parrish S, Imani F, Fire A and Morgan RA: Specific inhibition of gene expression by small double-stranded RNAs in invertebrate and vertebrate systems. Proc Natl Acad Sci USA 98: 9742-9747, 2001

23. Kumagai H, Tajima M, Ueno Y, Giga-Hama Y and Ohba M: Effect of cyclic RGD peptide on cell adhesion and tumor metastasis. Biochem Biophys Res Commun 177: 74-82, 1991.

24. Kaplan RN, Riba RD, Zacharoulis S, Bramley AH, Vincent L, Costa C, MacDonald DD, Jin DK, Shido K, Kerns SA, Zhu Z, Hicklin D, Wu Y, Port JL, Altorki N, Port ER, Ruggero D, Shmelkov SV, Jensen KK, Rafii S and Lyden D: VEGFR1positive haematopoietic bone marrow progenitors initiate the pre-metastatic niche. Nature 438: 820-827, 2005.

25. Sneath RJ and Mangham DC: The normal structure and function of CD44 and its role in neoplasia. Mol Pathol 51: 191200, 1998.
26. Bourguignon LY, Lokeshwar VB, Chen X and Kerrick WG: Hyaluronic acid-induced lymphocyte signal transduction and HA receptor (GP85/CD44)-cytoskeleton interaction. J Immunol 151: 6634-6644, 1993.

27. Bourguignon LY, Gilad E, Brightman A, Diedrich F and Singleton P: Hyaluronan-CD44 interaction with leukemiaassociated RhoGEF and epidermal growth factor receptor promotes Rho/Ras co-activation, phospholipase $\mathrm{C}$ epsilon- $\mathrm{Ca}^{2+}$ signaling, and cytoskeleton modification in head and neck squamous cell carcinoma cells. J Biol Chem 281: 14026-14040, 2006.

28. Bourguignon LY, Singleton PA and Diedrich F: HyaluronanCD44 interaction with Rac1-dependent protein kinase N-gamma promotes phospholipase Cgammal activation, $\mathrm{Ca}(2+)$ signaling, and cortactin-cytoskeleton function leading to keratinocyte adhesion and differentiation. J Biol Chem 279: 29654-29669, 2004.

29. Underhill C: CD44: the hyaluronan receptor. J Cell Sci 103: 293-298, 1992.

30. Dohadwala M, Batra RK, Luo J, Lin Y, Krysan K, Pold M, Sharma S and Dubinett SM: Autocrine/paracrine prostaglandin $\mathrm{E} 2$ production by non-small cell lung cancer cells regulates matrix metalloproteinase-2 and CD44 in cyclooxygenase-2-dependent invasion. J Biol Chem 277: 50828-50833, 2002.

31. Zhang Y, Thant AA, Machida K, Ichigotani Y, Naito Y, Hiraiwa Y, Senga T, Sohara Y, Matsuda S and Hamaguchi M: Hyaluronan-CD44s signaling regulates matrix metalloproteinase-2 secretion in a human lung carcinoma cell line QG90. Cancer Res 62: 3962-3965, 2002.

32. Yoshizaki T, Sato H and Furukawa M: Recent advances in the regulation of matrix metalloproteinase 2 activation: from basic research to clinical implication (Review). Oncol Rep 9: 607-611, 2002.

33. Li SS: Specificity and versatility of SH3 and other prolinerecognition domains: structural basis and implications for cellular signal transduction. Biochem J 390: 641-653, 2005.

34. Cohen GB, Ren R and Baltimore D: Modular binding domains in signal transduction proteins. Cell 80: 237-248, 1995.

35. Birge RB, Knudsen BS, Besser D and Hanafusa H: SH2 and SH3containing adaptor proteins: redundant or independent mediators of intracellular signal transduction. Genes Cells 1: 595-613, 1996.

36. Munaron L, Antoniotti S, Fiorio Pla A and Lovisolo D: Blocking $\mathrm{Ca}^{2+}$ entry: a way to control cell proliferation. Curr Med Chem 11: $1533-1543,2004$

37. Wang $L$ and Zhao B: The differences in some indexes of calcium homeostasis between the two types of ascites hepatoma cell line. Chin J Cancer 14: 265-267, 1995.

38. Martinez-Zaguilan R, Martinez GM, Gomez A, Hendrix MJ and Gillies RJ: Distinct regulation of pHin and $\left[\mathrm{Ca}^{2+}\right]$ in in human melanoma cells with different metastatic potential. J Cell Physiol 176: 196-205, 1998 .

39. Liu YQ, Gu B and Gao J: Measurement of the intracellular calcium of tumor cells with different metastatic potential and the effects of retinoamide. Chin J Pathophysiol 11: 259-262, 1995.

40. Morris JA, Kandpal G, Ma L and Austin CP: DISC1 (DisruptedIn-Schizophrenia 1) is a centrosome-associated protein that interacts with MAP1A, MIPT3, ATF4/5 and NUDEL: regulation and loss of interaction with mutation. Hum Mol Genet 12: 1591-1608, 2003

41. Goehler H, Lalowski M, Stelzl U, Waelter S, Stroedicke M, Worm U, Droege A, Lindenberg KS, Knoblich M, Haenig C, Herbst M, Suopanki J, Scherzinger E, Abraham C, Bauer B, Hasenbank R, Fritzsche A, Ludewig AH, Bussow K, Coleman SH, Gutekunst CA, Landwehrmeyer BG, Lehrach H and Wanker EE: A protein interaction network links GIT1, an enhancer of huntingtin aggregation, to Huntington's disease. Mol Cell 15: 853-865, 2004 